\title{
Os aspectos da sexualidade do idoso e os seus efeitos na qualidade de vida
}

\author{
The aspects of the sexuality of the elderly and its effects on the quality of life \\ Los aspectos de la sexualidad del mayor y sus efectos en la calidad de vida
}

Paula Cristina Nunes Nascimento ${ }^{1 *}$, Mayara Evangelista de Andrade $^{1}$, Maria de Fátima Ferreira Nóbrega ${ }^{1}$, Cleisla Thamires Lacerda Silva ${ }^{1}$, Nícia Stellita da Cruz Soares ${ }^{1}$, Lindomar de Farias Belém¹.

\section{RESUMO}

Objetivo: Investigar os fatores que podem interferir na sexualidade da população idosa e seus reflexos na Qualidade de Vida (QV). Métodos: Foi realizada uma pesquisa transversal com abordagem quantitativa, em um Programa de Ensino para Idosos na Paraíba. A partir de uma coleta de dados com a adaptação transcultural da Scale for Quality of Sexual Function (QSF) para português. O questionário do Google Forms foi enviado de forma online, devido ao distancimento social, ocasionado pela pandemia do covid-19. Posteriormente, foi realizado o processamento e digitação dos dados no Microsoft Excel e feita à análise dos dados no programa $R$ Core Team (2020). Resultados: Os achados da pesquisa sugerem que os fatores que interferem na sexualidade da população idosa, estão principalmente relacionados às questões hormonais, as quais refletem na redução de lubrificação, da resposta sexual, desejo e outros. Além disso, quesitos como idade elevada, sexo feminino e ausência parecem afetar ainda mais aos idosos. Conclusão: Conclui-se que os alunos da instituição estudada, são compostos majoritariamente por mulheres e grande parte dessas, podem sofrer interferências na QV, devido a alterações do envelhecimento, com um possível impacto mental e cognitivo.

Palavras-chave: Sexualidade, Idoso, Qualidade de vida.

\begin{abstract}
Objective: To investigate the factors that may interfere with the sexuality of the elderly population and their reflections on the Quality of Life (QOL). Methods: A cross-sectional research with a quantitative approach was carried out in a Teaching Program for the Elderly in Paraíba. From a data collection with the crosscultural adaptation of the Scale for Quality of Sexual Function (QSF) into Portuguese. The Google Forms questionnaire was sent online due to social distance caused by the covid-19 pandemic. Subsequently, data were processed and entered in Microsoft Excel and data were analyzed using the R Core Team program (2020). Results: The research findings suggest that the factors that interfere in the sexuality of the elderly population are mainly related to hormonal issues, which reflect in the reduction of lubrication, sexual response, desire and others. In addition, items such as old age, female gender and absence seem to affect even more the elderly. Conclusion: It is concluded that the students of the studied institution are mainly composed of women and a great part of them may suffer interference in the QoL, due to aging alterations, with a possible mental and cognitive impact.
\end{abstract}

Keywords: Sexuality, Aged, Quality of life.

\section{RESUMEN}

Objetivo: Investigar los factores que pueden interferir en la sexualidad de la población anciana y sus efectos en la Calidad de Vida (CV). Métodos: Se realizó una investigación transversal con enfoque cuantitativo en un Programa de Enseñanza para Adultos Mayores en Paraíba. De una recopilación de datos con la adaptación transcultural de la Escala de Calidad de la Función Sexual (QSF) al portugués. EI cuestionario de Google Forms se envió en línea debido a la distancia social causada por la pandemia del covid-19. Posteriormente, los datos fueron procesados e ingresados en Microsoft Excel y los datos fueron analizados usando el programa R Core Team (2020). Resultados: Los hallazgos de la investigación

1 Universidade Estadual da Paraíba (UEPB), Campina Grande - PB. *E-mail: paulacriscad26@gmail.com 
sugieren que los factores que interfieren en la sexualidad de la población anciana están relacionados principalmente con problemas hormonales, los cuales se reflejan en la reducción de la lubricación, respuesta sexual, deseo y otros. Además, elementos como la vejez, el sexo femenino y la ausencia parecen afectar aún más a las personas mayores. Conclusión: Se concluye que los estudiantes de la institución estudiada están compuestos mayoritariamente por mujeres y una gran parte de ellas pueden sufrir interferencias en la CV, por alteraciones del envejecimiento, con un posible impacto mental y cognitivo.

Palabras clave: Sexualidad, Anciano, Calidad de vida.

\section{INTRODUÇÃO}

O Brasil tem um histórico demográfico predominantemente composto de jovens, entretanto o seu perfil demográfico tem-se alterado expressivamente, visto a elevação da sobrevida dos brasileiros e consequentemente, um aumento na quantidade de pessoas idosas (QUEIROZ MAC, et al., 2015).

$\mathrm{Na}$ década passada era esperado que em 2020, houvesse uma grande quantidade de idosos, onde a maioria da população economicamente dependente seria maior do que a ativa. Tendo em vista, essa situação, percebe-se a primordialidade em considerar a saúde das pessoas idosas de maneira cada vez mais completa, incluindo o aspecto da sexualidade, o qual é muitas vezes desconsiderado por grande parcela da sociedade (ALENCAR DL, et al., 2014).

É inegável que ocorrem alterações importantes nessa faixa etária, dentre elas referentes ao corpo, como por exemplo, perda da musculatura; cabelos tornam-se esbranquiçados; a pele tem uma menor elasticidade; a perda dos dentes; o metabolismo torna-se mais lento; surgem problemas circulatórios, alterações de sentidos, mudanças fisiológicas e físicas do envelhecer. Embora aconteçam perdas, a pessoa idosa pode viver satisfatoriamente (QUEIROZ MAC, et al., 2015; VIEIRA KFL, et al., 2016).

Para Viera KFL, et al. (2016) o envelhecer é um processo de muita complexidade e singularidade, e não quer dizer obrigatoriamente que haverá dependência, inutilidade funcional, ou que o individuo deixará de viver situações em sociedade, inclusive a sexualidade.

A sexualidade é inerente ao individuo, isto é, está presente durante toda vida, sendo esta, marcada como uma forma específica e característica de sentir, se expressar e se comunicar, advindos desde a fase infantil até a terceira idade (ALENCAR DL, et al., 2016).

Este aspecto de vida acontece através de interações com o outrem e é exteriorizada durante as relações em sociedade, por meio do corpo, ou da forma de agir no mundo. Diferindo do ato sexual, pois o sexo retrata apenas uma forma de expressão desta. Ela faz parte da totalidade da pessoa e deve ser observada de forma biopsicossocial. Mas, quando se refere à sexualidade de idosos, a população em geral, considera que essa faixa etária é assexuada e são disseminados preconceitos, mitos, tabus, entre outras questões (UCHÔA YS, et al., 2016; ALENCAR DL, et al., 2014).

Neste enfoque, a sexualidade está presente ainda no domínio de percepção individual da qualidade de vida. Esta é conceituada pela Organização Mundial da Saúde (OMS), como o modo com que a pessoa enxerga a sua existência, em seus aspectos culturais, além de valores, expectativas e objetivos pessoais (UCHÔA YS, et al., 2016).

Conceitos errôneos, criados em relação aos idosos, podem resultar em danos a qualidade de vida. $O$ profissional da saúde, inclusive o enfermeiro, deve promover educação, a partir de análise de condições que podem levar a essas definições preconceituosas que interferem no aspecto sexual dessa faixa etária (VIEIRA KFL, et al., 2016; ALENCAR DL, et al., 2014).

Segundo Kuawe CA, et al. (2015) a Universidade Aberta à terceira idade também é um local de convivência em sociedade que promove qualidade de vida, pois é um espaço de estimulação a atividades artísticas, físicas e a aquisição de novos conhecimentos.

Os programas de Universidades para idosos visam proporcionar a pessoa idosa, melhor qualidade de vida, corroborando assim, para aspectos sociais, particulares e funcionais, tendo em vista, a dinamização e 
criação de atividades constantes. Estas atividades objetivam a inserção do idoso no ambiente acadêmico, em atividades culturais, ações socioeducativas, estímulo à autonomia, entre outros. Além disso, promover o envelhecimento ativo ajuda na autoestima destes (LIMA RA, et al., 2017).

Desta forma o trabalho justifica-se, pois, a sexualidade está presente em todas as fases da vida, ou seja, interfere no aspecto de qualidade de vida, podendo-se observar essa questão em alunos do programa de ensino para idosos, sendo este, um local de interação e quebra de paradigmas relacionados à saúde da pessoa idosa. Por fim, objetiva investigar os fatores que podem interferir na sexualidade da população idosa e seus reflexos na Qualidade de Vida (QV).

\section{MÉTODOS}

Foi realizada uma pesquisa transversal com abordagem quantitativa, em um Programa de Ensino para Idosos na Paraíba. A população abordada foram os idosos matriculados e formados no ano de 2019, tendo uma amostra de 27 pessoas, sendo 12 matriculados no ano de 2020 e 15 formados no ano anterior.

A coleta de dados foi feita com a adaptação transcultural da Scale for Quality of Sexual Function (QSF) para português, instrumento que investiga a atividade sexual em ambos os sexos por autoaplicação, em seu conteúdo apresenta 32 questões, sendo oito genéricas e os outros singulares. Os itens dividem-se os seguintes domínios: qualidade de vida psicossomática, Atividade Sexual, (Dis)função Sexual - autorreflexão e (Dis)função Sexual - visão do parceiro (PEREIRA, VM, et al., 2011). O questionário foi respondido de forma virtual no Google Forms, visto que o local de ensino está fechado devido à pandemia do covid-19.

As variáveis do estudo são as seguintes, independentes: características sociodemográficas; e sexualidade; e variável dependente: qualidade de vida. Para o processamento e digitação, os dados coletados foram colocados no programa EXCEL, sendo este o banco de dados, o qual teve digitação dupla e sistematização destes.

No que tange a análise de dados utilizou-se o programa $R$ Core Team (2020), para realizar a análise estatística descritiva. A análise foi fundamentada em "sexualidade no idoso" e "qualidade de vida". Os resultados foram dispostos em gráficos e tabelas mostrando os dados relativos às categorias: qualidade de vida psicossomática, Atividade Sexual e (Dis)função Sexual (autorreflexão e visão do parceiro).

Em relação aos aspectos éticos segui a resolução no 466 , de 12 de dezembro de 2012, a qual discorre a cerca de aspectos éticos das pesquisas com seres humanos sempre respeitando a dignidade, liberdade e autonomia deles (BRASIL, 2012).

O estudo foi enviado ao comitê de ética da Paraíba, de acordo com o Parecer Consubstanciado de número 3.974.258, e com o Certificado de Apresentação e Apreciação Ética (CAAE) de número 29942819.3.0000.5187. Após a aprovação, foi entregue a cada individuo um Termo de Consentimento Livre e Esclarecido (TCLE), de forma virtual.

\section{RESULTADOS E DISCUSSÃO}

No estudo, foram incluídos 27 participantes, entre os quais $74,07 \%$ (20) eram mulheres. Isto corrobora com o fenômeno chamado feminização do envelhecimento, que é caracterizado pela maior quantidade de mulheres idosas em relação aos homens (SOUSA NFS, et al., 2018).

No entanto, apesar das mulheres viverem mais, elas têm pior qualidade de vida, quando é feita a comparação com os homens, visto que existem inúmeras diferenciações entre homens e mulheres, sobretudo na visão social, como menores benefícios nas condições de trabalho em geral, além disso, os homens têm muita dificuldade no que diz respeito ao autocuidado, o que os torna mais vulneráveis. Além disso, estudos mostram que o público feminino em programas de universidade para pessoas idosas tem um número muito superior do que o masculino (SOUSA NFS, et al., 2018; ADAMO CE, et al., 2017).

De acordo com os dados encontrados, verifica-se que a altura média do público feminino foi de $1,56 \mathrm{~m}$, com pouca variabilidade coeficiente de variação $(\mathrm{CV})<10 \%$, bem como nos homens foi de $1,70 \mathrm{~m}$ com 
variabilidade pequena, $\mathrm{CV}<10 \%$, ou seja, os valores absolutos das alturas relação à média foram pequenos. Em relação aos pesos e as idades essa média foi de $65,50 \mathrm{~kg}$ e 66,30 anos no sexo feminino, enquanto no sexo masculino foi de $74,43 \mathrm{~kg}$ e 68,29 anos com média e baixa variabilidade, $10 \%<\mathrm{CV}<20 \%$ e CV $<10 \%$, em ambos, ou seja, relativo a média os valores absolutos dos pesos e das idades foram pequenos (Tabela 1).

Tabela 1 - Idades, Pesos e Alturas dos Idosos do Programa de Ensino.

\begin{tabular}{llllllllll}
\hline Sexo & ASD & Média & Mín & Máx & $\mathbf{n}$ & Me & Mo & D.P & CV(\%) \\
\hline \multirow{3}{*}{ Feminino } & Idade (anos) & 66,30 & 60,00 & 85,00 & 20 & 65,00 & 65,00 & 5,97 & 9,01 \\
& Peso $(\mathrm{Kg})$ & 65,50 & 54,00 & 93,00 & 20 & 64,00 & 65,00 & 8,78 & 13,40 \\
& Altura $(\mathrm{m})$ & 1,56 & 1,47 & 1,65 & 20 & 1,54 & $(1,53$ e 1,60) & 0,05 & 3,41 \\
\hline \multirow{2}{*}{ Sexo } & ASD & Média & Mín & Máx & $\mathbf{n}$ & Me & Mo & D.P & CV(\%) \\
\hline \multirow{3}{*}{ Masculino } & Idade (anos) & 68,29 & 63,00 & 74,00 & 7 & 67,00 & 74,00 & 4,68 & 8,75 \\
& Peso $(\mathrm{Kg})$ & 74,43 & 59,00 & 85,00 & 7 & 76,00 & 80,00 & 8,73 & 11,73 \\
& Altura $(\mathrm{m})$ & 1,70 & 1,63 & 1,76 & 7 & 1,70 & 1,70 & 0,04 & 2,62 \\
\hline
\end{tabular}

Legenda: ASD: Aspectos Sócio - Demográficos; Mín: Mínimo das observações; Máx: Máximo das observações; n: Tamanho da amostra; Me: Mediana das observações; Mo: A moda das observações; D.P: Desvio Padrão das observações; $\mathrm{CV}(\%)$ : Coeficiente de variação das observações.

Fonte: Nascimento PCN, et al., 2021.

Outros dados encontrados foram referentes ao domínio da QV na visão psicossomática. Considera-se como fenômeno psicossomático algo que advém do campo mental para o orgânico. Este fenômeno afeta a QV, além de que, de acordo com a psicanálise, neste é mantida a lei do desejo (CERCHIARI EAN, 2000; RINALDI D, et al., 2013).

Nesta perspectiva, os sintomas mais mencionados foram irritabilidade e nervosismo aumentado (70\%); humor deprimido (67\%); memória e concentração prejudicadas (67\%); força muscular diminuída (59\%); e dor muscular e articular (82\%).

Concernente à irritabilidade e nervosismo a quantidade de pessoas que relataram esse fator aumentado foi $33 \%$ leve, $26 \%$ moderado, $7 \%$ grave e $4 \%$ muito grave. Enquanto, no humor deprimido se observou que $33 \%$ das pessoas idosas não se sentem deprimidos, mas a grande maioria relatou este fator de leve a muito grave.

Corroborando a estes achados, outra pesquisa Menezes DV e Oliveira ME (2016) encontraram resultados significativos, relacionados a estes sintomas de forma moderada em pessoas do sexo feminino e sugeriram a ocorrência de possível surgimento de algum tipo de transtorno mental, como depressão e/ou ansiedade. Além disso, em um estudo distinto, também foi percebida uma correlação com as mulheres, relativas às mudanças hormonais ocorridas no climatério, juntamente com a diminuição da autoestima destas, em comparação aos homens, podendo ainda afetar na $Q V$ e atividade sexual do individuo (SKOPINSKI F, et al., 2015; LORENZI DRS e SACILOTO B, 2006).

No tocante a memória e concentração prejudicadas, mais da metade referiram a positividade deste fator em sua vida de forma leve, enquanto apenas $15 \%$ proferiu ter este de moderado a muito grave. Um estudo oposto identificou que queixas relativas à memória, são comuns em idosos e pode está relacionada à mudança de humor e eleva as chances de se ter demência. Além disso, mesmo que em frequência menor, também foi um sintoma relacionado à piora da QV (BRIGOLA AG, et al., 2015).

Quanto à dor obteve-se um resultado de $82 \%$, para a presença deste elemento de forma leve e moderada, desses apenas $19 \%$, não sentem nenhuma dor muscular ou nas articulações. O estudo de Sobrinho ACS, et al., (2019), reforça este resultado, no qual foi encontrado em seus achados que $89,22 \%$ dos pesquisados apresentavam dor, além de que se relacionou a dor com o aumento do nível de estresse. Foi sugerido anteriormente ainda, que na medida em que ocorre o envelhecimento, os receptores dos sensores dolorosos do individuo tem uma diminuição funcional. Todavia, a população idosa, geralmente acredita de forma equivocada que a dor é algo natural do processo de envelhecer. 
No que diz respeito à força muscular $41 \%$ da população relatou não haver nenhuma diminuição da força muscular, embora 37\% explanem ter uma queda leve, e 15\% de forma moderada. Uma pesquisa constatou que a diminuição da força muscular, sobretudo do assoalho pélvico pode aumentar as chances de comprometer a função sexual (KRÜGER AP, et al., 2012).

No tocante aos aspectos relacionados à sexualidade, como a presença do parceiro na qual se constatou que $33,33 \%$ (9) das pessoas têm, dentre estes, 18,52\% (5) são mulheres. Dessas, 25,92\% (7) tiveram atividade sexual no último mês, sendo $14,81 \%$ (4) do sexo feminino. Com relação ao tempo de intimidade, $18,52 \%$ (5) deles apresentavam um período maior que de 10 anos, enquanto outros $14,81 \%$ (4) menor do que 6 meses.

Sendo assim, percebe-se que pequena parte da população pesquisada tem parceiro e grande parte das pessoas acompanhadas, tiveram relações sexuais no mês anterior à realização da pesquisa. Além disso, vê-se que a maioria das pessoas que tem companheiro(a) tem uma intimidade há mais de dez anos, enquanto, pouco menos da metade tem há seis meses, o qual é considerado pouco tempo, quando são comparados.

Isto valida os resultados de outro estudo, no qual apresentou que presença de parceiro(a) afetivo(a), pode levar ao aumento da frequência de atividade sexual, além de que os indivíduos considerados ativos mantêm o desejo sexual (RODRIGUES LR, et al., 2018).

Outrossim, de acordo com a Classificação Internacional de doenças da OMS, conceitua-se a disfunção sexual, como a incapacidade de uma pessoa participar de um ato sexual, através de vários aspectos. Além disso, esta abrange fenômenos psicossomáticos e/ ou físicos (CARRILHO PJF, et al., 2015).

Nos homens, esta disfunção está muito relacionada à Disfunção Erétil (DE), a qual é definida como a incapacidade de ereção do órgão genital masculino. Este fator está relacionado a inúmeras questões psíquicas e comportamentais (RIBEIRO JP e SANTOS A, 2005).

Enquanto, nas mulheres esta disfunção sexual é um dos sintomas do período da menopausa, sendo esta uma dificuldade a qual incluí aspectos tanto psicológicos como orgânicos, levando a consequências na qualidade de vida de pessoas do sexo feminino após esse período. Corroborando com isso, outra pesquisa mostrou que $90 \%$ das mulheres com disfunção sexual tinham também depressão (MENEZES DV e OLIVEIRA ME, 2016; SREELAKSHMY K, et al., 2017).

No tocante ao desejo sexual, relacionado ao domínio da (dis)função na autorreflexão, foi verificado que $35 \%$ do público feminino e $86 \%$ do público masculino alegaram terem desejo de levemente a muito fortemente. Além disso, $25 \%$ das mulheres relataram que não gostaria de ter contatos sexuais com maior frequência, enquanto os indivíduos do sexo masculino, não relataram a falta de desejo (Tabela 2).

Com respeito ao mesmo aspecto apresentado anteriormente, porém na visão dos idosos estudados, referente aos seus parceiros, se obteve um percentual de $35 \%$, em que o sexo feminino alegou que os parceiros têm interesse sexual (de levemente a moderadamente) e 14\% do percentual masculino alegaram que suas parceiras gostariam de ter contatos sexuais moderadamente mais frequentes. Sendo assim, infere-se que este aspecto, está mais presente no sexo masculino, não só no domínio da autorreflexão, como na visão das mulheres sobre os seus companheiros.

Por conseguinte, este achado pode ser explicado por outro artigo, pela questão hormonal nas mulheres em período de pós-menopausa, além de diminuição da autoestima que pode ocorrer neste momento da vida da mulher (SKOPINSKI F, et al., 2015).

Conquanto, é necessário considerar que este desejo está envolvido a diversos fatores imensuráveis, dentre eles, espirituais, sociais, psicológicos, biológicos e históricos. Além de que os achados da pesquisa sugerem ainda outros aspectos que interferem neste fator, principalmente ligados ao relacionamento, como, poder do homem sobre a parceira, questões culturais e incompreensão do parceiro. Outro aspecto pertinente é a excitação sexual, em que se percebeu que apenas 18,52\% (5) pessoas afirmaram que experimentam moderadamente de grande excitação sexual antes e durante a relação sexual, sendo 7,40\% 
(2) do sexo feminino e $11,11 \%$ (3) do sexo masculine. Este achado pode ser apoiado por um estudo anterior, que identificou a diminuição da libido, entretanto, relacionado à menor quantidade do hormônio testosterona devido o processo de envelhecimento (VALENÇA CN, et al., 2010).

Tabela 2 - (Dis)função sexual (Autorreflexão e visão do parceiro): Desejo sexual.

\begin{tabular}{lcccc}
\hline $\begin{array}{l}\text { Desejo de maior frequência sexual } \\
\text { (Autorreflexão) }\end{array}$ & $\begin{array}{c}\text { Feminino } \\
\text { Frequência } \\
\text { (Absoluta) }\end{array}$ & $\begin{array}{c}\text { Percentual } \\
\text { (\%) }\end{array}$ & $\begin{array}{c}\text { Masculino } \\
\text { (Absoluta) }\end{array}$ & $\begin{array}{c}\text { Percentual } \\
\text { (\%) }\end{array}$ \\
\hline Fortemente & 1 & 5 & 2 & 29 \\
Levemente & 3 & 15 & 3 & 43 \\
Moderadamente & 2 & 10 & 0 & 0 \\
Muito fortemente & 1 & 5 & 1 & 14 \\
Não & 5 & 25 & 0 & 0 \\
Sem Parceiro & 8 & 40 & 1 & 14 \\
\hline Desejo de maior frequência sexual & Frequência & Percentual & Frequência & Percentual \\
(Visão do parceiro) & (Absoluta) & $(\%)$ & (Absoluta) & $(\%)$ \\
\hline Fortemente & 1 & 5 & 0 & 0 \\
Levemente & 4 & 20 & 0 & 0 \\
Moderadamente & 2 & 10 & 1 & 14 \\
Não & 3 & 15 & 3 & 43 \\
Sem Parceiro & 10 & 50 & 3 & 43 \\
\hline
\end{tabular}

Fonte: Nascimento PCN, et al., 2021.

Correlacionado a isto, está à resposta sexual, onde 25,92\% (7) pessoas afirmaram que seus órgãos sexuais respondem moderadamente a desejos sexuais ou sonhos eróticos, sendo $14,81 \%$ (4) do sexo masculino. Enquanto, que 18,52\% (5) pessoas afirmaram que a resposta sexual acontece frequentemente, sendo $11,11 \%$ (3) do sexo feminino. Comprovando esta pesquisa, uma anterior realizada por Santos; Santos; Cendoroglo, (2015), encontraram que a maioria das pessoas tinha níveis baixos de estimulação, no que tange a capacidade de resposta sexual. No quesito lubrificação apenas $14,81 \%$ (4) pessoas afirmaram que alcançam frequentemente a lubrificação necessária durante a relação sexual, sendo dessas $11,11 \%$ (3) do sexo masculino. Entretanto, 33,33\% (9) pessoas afirmaram não alcançar esse estágio, desses, 14,81\% (4) eram mulheres. Percebe-se também que as mulheres tem uma menor lubrificação quando comparadas aos homens.

Com relação à dispaurenia, $30 \%$ das mulheres e $43 \%$ dos homens, alegaram que não apresentavam. No entanto, $14 \%$ das pessoas do sexo masculino alegaram que sentiam dor levemente e $20 \%$ do sexo feminino de leve a fortemente durante a relação sexual. Enquanto, os indivíduos do sexo feminino alegaram que $50 \%$ dos seus parceiros não sentiam nenhum problema durante a relação sexual, e neste mesmo aspecto, foi alegado que $43 \%$ das parceiras do sexo masculino, também não apresentavam dispareunia (Tabela 3).

Tabela 3 - (Dis)função sexual (Autorreflexão e visão do parceiro): Dispareunia.

\begin{tabular}{lcccc}
\hline & Feminino & Masculino & \\
\hline $\begin{array}{l}\text { Dispareunia } \\
\text { (Autorreflexão) }\end{array}$ & $\begin{array}{c}\text { Frequência } \\
\text { (Absoluta) }\end{array}$ & $\begin{array}{c}\text { Percentual } \\
(\%)\end{array}$ & $\begin{array}{c}\text { Frequência } \\
\text { (Absoluta) }\end{array}$ & $\begin{array}{c}\text { Percentual } \\
(\%)\end{array}$ \\
\hline Fortemente & 1 & 5 & 0 & 0 \\
Levemente & 2 & 10 & 1 & 14 \\
Moderadamente & 1 & 5 & 0 & 0 \\
Não & 6 & 30 & 3 & 43 \\
Sem Parceiro & 10 & 50 & 3 & 43 \\
\hline Dispareunia (visão do & Frequência & Percentual & Frequência & Percentual \\
parceiro) & (Absoluta) & $(\%)$ & 0 & $(\%)$ \\
\hline Levemente & 1 & 5 & 1 & 0 \\
Muito fortemente & 0 & 0 & 3 & 44 \\
Não & 10 & 50 & 3 & 43 \\
Sem Parceiro & 9 & 45 & & \\
\hline
\end{tabular}

Fonte: Nascimento PCN, et al., 2021. 
Algumas pesquisas apontam que o ressecamento vaginal gerado pela diminuição da lubrificação é um fator sugestivo para o aparecimento da dispareunia (MENEZES DV e OLIVEIRA ME, 2016; VALENÇA CN, et al., 2010). Posto isso, a partir dos achados, se vê que apesar de uma quantidade pequena das mulheres pesquisadas não apresentarem lubrificação suficiente, uma quantidade aproximada do sexo feminino apresenta a dor durante a relação sexual, embora as que sintam este sintoma sejam de forma leve a forte.

Dentre os 27 participantes, apenas $22,2 \%$ (6) afirmaram que alcançam frequentemente o orgasmo durante a relação sexual, sendo $11,11 \%$ (3) do sexo feminino e a mesma quantidade do sexo masculine. Considerando-se assim, que foi uma porcentagem pequena que alcançou este ponto sexual. Outras pesquisas mostraram que dentre as disfunções sexuais mais presentes em idosos, está o alcance do orgasmo (SANTOS AM, et al., 2015).

Após a compreensão de fatores que podem interferir no ato sexual, é pertinente salientar que a sexualidade vai, além disso, sendo assim, torna-se pertinente salientar que esta não está relacionada apenas ao ato físico, mas envolve aspectos biológicos, sociais e psicológicos, sendo importante compreender a totalidade (RODRIGUES LR, et al., 2018).

Posto isto, a presença de sonhos eróticos, fantasias ou desejos sexuais, também é um aspecto da sexualidade, neste sentido, verifica-se que foi alegado por $40 \%$ do público feminino e $57 \%$ do público masculino que tem a presença de sonhos deste tipo, de raramente a muito frequentemente. Em relação a seus parceiros, $20 \%$ do sexo feminino alegaram que os seus parceiros tinham sonhos eróticos de raramente a moderadamente, enquanto os homens relataram que as parceiras não tinham a presença deste elemento (Tabela 4).

Tabela 4 - (Dis)função sexual (Autorreflexão e visão do parceiro): sonhos eróticos, fantasia ou desejo sexual.

\begin{tabular}{lcccc}
\hline $\begin{array}{l}\text { Sonhos eróticos e outros } \\
\text { (Autorreflexão) }\end{array}$ & $\begin{array}{c}\text { Feminino } \\
\text { (Absoluta) }\end{array}$ & $\begin{array}{c}\text { Percentual } \\
(\%)\end{array}$ & $\begin{array}{c}\text { Masculino } \\
\text { Frequência } \\
\text { (Absoluta) }\end{array}$ & $\begin{array}{c}\text { Percentual } \\
(\%)\end{array}$ \\
\hline Frequentemente & 1 & 5 & 0 & 0 \\
Moderadamente & 2 & 10 & 1 & 14 \\
Muito frequentemente & 2 & 10 & 1 & 14 \\
Não & 9 & 45 & 3 & 43 \\
Raramente & 3 & 15 & 2 & 29 \\
Sem Parceiro & 3 & 15 & 0 & 0 \\
\hline Sonhos eróticos e outros & Frequência & Percentual & Frequência & Percentual \\
(visão do parceiro) & (Absoluta) & $(\%)$ & (Absoluta) & $(\%)$ \\
\hline Moderadamente & 2 & 10 & 0 & 0 \\
Não & 7 & 35 & 4 & 57 \\
Raramente & 2 & 10 & 0 & 0 \\
Sem Parceiro & 9 & 45 & 3 & 43 \\
\hline
\end{tabular}

Fonte: Nascimento PCN, et al., 2021.

Dessa forma, é valido salientar o pensamento de Freud S (1980) apud Zavaroni DML (2007), o qual diz que os sonhos são caracterizados como uma forma de acessar a vivencias que foram recalcadas e que não poderiam mais ser lembradas. Seguindo este pensamento, pode-se dizer que a presença de sonhos eróticos, pode significar o recalque do desejo sexual.

A autossatisfação sexual, também é outra forma de exercer a sexualidade e quanto aos achados relativos a este domínio tem-se que $37,03 \%$ (10) pessoas afirmaram que não se autossatisfazem sexualmente, sendo $33,33 \%$ (9) do sexo feminino. Enquanto apenas 22,22\% (6) pessoas afirmaram que pouco se autossatisfazem, sendo $11,11 \%$ (3) do sexo feminino e a mesma quantidade do sexo masculino. Um estudo diferente mostrou um resultado que contribuiu a este, onde $24 \%$ relataram o autoerotismo (RODRIGUES LR, et al., 2018).

Somado a isto, diversos autores relatam que há uma diminuição significativa da autossatisfação sexual de acordo com a elevação da idade, porém a sexualidade não se reduz de forma muito expressiva (SANTOS AM, et al., 2015). 
De acordo com os achados, tem-se que $44,44 \%$ (12) pessoas afirmaram que a sexualidade tem um papel importante em sua vida, sendo $25,92 \%$ (7) pessoas do sexo feminino e $18,51 \%$ (5) pessoas do sexo masculino. Enquanto $37,03 \%$ (10) pessoas afirmaram que a sexualidade tem um papel pouco importante em sua vida, sendo 33,33\% (9) pessoas do sexo feminino e 3,7\% (1) pessoas do sexo masculino.

Outra pesquisa mostrou que a maior parte da população pesquisada, acredita na importância da sexualidade na qualidade de vida. Dessa forma, torna-se essencial entender como os idosos vivenciam este momento e como são as suas percepções, neste aspecto (RODRIGUES LR, et al., 2018).

Percebe-se que as mulheres tem muito mais fatores que podem interferir na função sexual, e consequentemnte na sexualidade em geral e refletir na qualidade de vida do que os homens, principalmente, pela questão hormonal, mas também relativas a questões sociais, culturais e psicológicas, nas quais, se tem todo um contexto histórico e de submissão que pode interferir.

Este trabalho apresenta algumas limitações, tais como o tamanho da amostra, tipo de coleta (Online), considerando a população abordada, impossibilitando a generalização do mesmo. Posto isso, se faz necessária a realização de mais pesquisas com amostras maiores, para que se possa haver um estabelecimento de prevalência desses aspectos na população idosa com maior robustez.

\section{CONCLUSÃO}

A maioria da população idosa do programa de ensino para idosos é do sexo feminino, além disso, grande parte desta população não tem um parceiro afetivo, o que pode levar a diminuição da frequência sexual, outros fatores também corroboram a essa redução, como diminuição do desejo sexual, da lubrificação, da resposta sexual, dentre outras condições, percebidas principalmente nas mulheres, que podem gerar impacto na sexualidade. Ainda neste contexto, relativo aos sonhos parece estar mais presente em homens, ao passo que a autossatisfação sexual se apresenta de forma igual entre os dois sexos. Além disso, percebe-se impacto na QV, devido ao envelhecimento, referente aos aspectos mentais, cognitivos e sexuais.

\section{REFERÊNCIAS}

1. $A D A M O C E$, et al. Universidade aberta para a terceira idade: 0 impacto da educação continuada na qualidade de vida dos idosos. Revista Brasileira de Geriatria e Gerontologia, 2017; $20(04): 545-555$.

2. ALENCAR DL, et al. Exercício da sexualidade em pessoas idosas e os fatores relacionados. Revista Brasileira de Geriatria e Gerontologia, 2016; 19(5): 861-869.

3. ALENCAR DL, et al. Fatores que interferem na sexualidade de idosos: uma revisão integrativa. Ciência \& Saúde Coletiva, 2014; 19(8): 3533-3542.

4. BRASIL. Ministério da Saúde. Conselho Nacional de Saúde. Resolução oㅡ 466, de 12 de dezembro de 2012. Aprova diretrizes e normas regulamentadoras de pesquisas envolvendo seres humanos. Brasília, Diário Oficial da União, 12 dez. 2012.

5. BRIGOLA AG, et al. Subjective memory complaints associated with depression and cognitive impairment in the elderly: a systematic review. Dementia \& Neuropsychologia, 2015; 9(1): 51-57.

6. CARRILHO PJF, et al. Sexual dysfunction in obese women is more affected by psychological domains than that of non-obese. Revista Brasileira de Ginecologia e Obstetricia, 2015; 37(12): 552-558.

7. CERCHIARI, EAN. Psicossomática um estudo histórico e epistemológico. Psicologia: ciência e profissão, 2000; 20(4): 64-79.

8. KRÜGER AP, et al. Função muscular do assoalho pélvico e função sexual em mulheres segundo a faixa etária e os níveis de atividade física. Dissertação (Mestrado em Ciências do Movimento Humano). Universidade do Estado de Santa Catarina, Santa Catarina, Florianópolis, 2012; 97 p.

9. KUWAE CA, et al. Concepções de alimentação saudável entre idosos na Universidade Aberta da Terceira Idade da UERJ: normas nutricionais, normas do corpo e normas do cotidiano. Revista Brasileira de Geriatria e Gerontologia, 2015; 18(3): 621-630.

10. LIMA RA, et al. Universidade Aberta à Maturidade-UEPB: Oito anos de educação inclusiva e transformadora. Campina Grande: EDUEPB, 2017; 134p.

11. LORENZI DRS, SACILOTO B. Freqüência da atividade sexual em mulheres menopausadas. Revista da Associação Médica Brasileira, 2006; 52(4): 256-260. 
12. MENEZES DV, OLIVEIRA ME. Avaliação da qualidade de vida de mulheres no climatério na cidade de Floriano, Piauí. Fisioterapia em Movimento, 2016; 29(2): 219-227.

13. PEREIRA VM, et al. Tradução e adaptação transcultural para o português brasileiro da Scale for Quality of Sexual Function (QSF). Revista de Psiquiatria do Rio Grande do Sul, 2011; 33(2): 87-97.

14. QUEIROZ MAC, et al. Representações sociais da sexualidade entre idosos. Revista brasileira de enfermagem, 2015 ; 68(4): 662-667.

15. R Core Team (2020). R: A language and environment for statistical computing. R Foundation for statistical computing, Vienna, Autria. URL https://www.R-project.org/.

16. RIBEIRO JP, SANTOS A. Estudo exploratório da relação entre função eréctil, disfunção eréctil e qualidade de vida em homens portugueses saudáveis. Análise Psicológica, 2005; 23(3): 341-349.

17. RINALDI D, et al. Do fenômeno psicossomático ao sintoma: a aderência do sujeito ao diagnóstico médico e o trabalho analítico. Ágora: Estudos em Teoria Psicanalítica, 2013; 16 (spe): 95-108.

18. RODRIGUES LR, et al. Análise do comportamento sexual de idosas atendidas em um ambulatório de ginecologia. Revista Brasileira de Geriatria e Gerontologia, 2018; 21(6): 724-730.

19. SANTOS AM, et al. Sexuality and chronic pain in long-lived females: description of interferential factors. Revista Dor, $2015 ; 16(1): 48-52$.

20. SKOPINSKI F, et al. Imagem corporal, humor e qualidade de vida. Revista Brasileira de geriatria e gerontologia, 2015; 18(1): 95-105.

21. SOBRINHO ACS, et al. Associação de dor crônica com força, níveis de estresse, sono e qualidade de vida em mulheres acima de 50 anos. Fisioterapia e Pesquisa, 2019; 26(2): 170-177.

22. SOUSA NFS, et al. Envelhecimento ativo: prevalência e diferenças de gênero e idade em estudo de base populacional. Cadernos de Saúde Pública, 2018; 34(11): e00173317.

23. SREELAKSHMY K, et al. Sexual dysfunction in females with depression: a cross-sectional study. Trends in psychiatry and psychotherapy, 2017; 39(2): 106-109.

24. UCHÔA YS, et al. A sexualidade sob o olhar da pessoa idosa. Revista Brasileira de Geriatria e Gerontologia, 2016; 19(6): 939-949.

25. VALENÇA CN, et al. Mulher no climatério: reflexões sobre desejo sexual, beleza e feminilidade. Saúde e Sociedade, 2010; 19(2): 273-285.

26. VIEIRA, KFL, et al. A sexualidade na velhice: representações sociais de idosos frequentadores de um grupo de convivência. Psicologia: ciência e profissão, 2016; 36(1): 196-209.

27. ZAVARONI DML, et al. A constituição do infantil na obra de Freud. Estudos de Psicologia (Natal), 2007; 12(1): 65-70. 